Homology, Homotopy and Applications, vol.6(1), 2004, pp.473-500

\title{
EQUIVARIANT COVERING SPACES AND HOMOTOPY COVERING SPACES
}

\author{
STEVEN R. COSTENOBLE AND STEFAN WANER \\ (communicated by Gunnar Carlsson)
}

\begin{abstract}
Nonequivariantly, covering spaces over a connected (locally nice) space $X$ are in one-to-one correspondence with actions of the fundamental group of $X$ on discrete sets. For nonconnected spaces we consider instead actions of the fundamental groupoid. In this paper we generalize to the equivariant case, showing that we can use either of two possible notions of action of the equivariant fundamental groupoid. We consider both equivariant covering spaces and the more general notion of equivariant homotopy covering spaces.
\end{abstract}

\section{Introduction}

The theory of equivariant classifying spaces gives us a homotopy representation of covering spaces (and other fibrations), but not an algebraic representation. In this paper we give a long overdue algebraic classification of equivariant covering spaces and homotopy covering spaces, analogous to the classical nonequivariant results based on the fundamental group.

Unless stated otherwise, $G$ will be a compact Lie group and all subgroups will be understood to be closed. By an equivariant covering space we shall mean the following, which is slightly more general than the usual meaning of covering space. Recall that a $G$-fibration is a $G$-map having the $G$-covering homotopy property [14].

Definition 1.1. A $G$-covering space is a $G$-fibration $p: E \rightarrow B$ with discrete fibers.

Note that this agrees with the usual notion of covering space when $B$ is locally nice, for example locally contractible.

Nonequivariantly, the category of covering spaces is equivalent to the category of fibrations with homotopy discrete fibers and fiber-homotopy classes of maps, under suitable point-set topological assumptions. This follows from the fact that we can replace each homotopy discrete fiber with its discrete set of components. However, equivariantly there are at least two reasonable meanings for the term "homotopy discrete." One would be a $G$-space $G$-homotopy equivalent to a discrete $G$-set; another would be a $G$-space each of whose fixed-sets is homotopy equivalent to a

Received November 15, 2002, revised October 25, 2004; published on December 28, 2004.

2000 Mathematics Subject Classification: Primary 55R91; Secondary 18B40, 22A30, 55N25, 55N91, $55 \mathrm{R} 15$.

Key words and phrases: Covering spaces, equivariant homotopy theory.

(C) 2004, Steven R. Costenoble and Stefan Waner. Permission to copy for private use granted. 
discrete set. The first of these notions leads to nothing significantly different from the nonequivariant case; the second notion is more interesting and needed in a variety of applications, including the definition of equivariant homology or cohomology with local coefficients [2] (see also the last section of this paper). Examples of homotopy discrete $G$-spaces in the latter sense go back Palais's universal $G$-spaces [9] (see also $[4]$ for an elegant construction and generalizations). Here are the formal definitions.

Definition 1.2. A $G$-space $F$ is (weakly) homotopy discrete if, for every subgroup $K$ of $G$, the discretization map $F^{K} \rightarrow \pi_{0}\left(F^{K}\right)$ is continuous and a (weak) homotopy equivalence. To be a homotopy discrete space, we also insist that $F$ have the $G$ homotopy type of a $G$-CW complex.

Note that the discretization map $F^{K} \rightarrow \pi_{0}\left(F^{K}\right)$ is continuous if and only if $F^{K}$ is locally path-connected.

Definition 1.3. A (weak) homotopy $G$-covering space is a $G$-fibration $p: E \rightarrow B$ such that, for each $b \in B$, the fiber $p^{-1}(b)$ is a (weakly) homotopy discrete $G_{b}$-space.

Definition 1.4. Let $\mathcal{C}(X)$ denote the category of $G$-covering spaces over $X$ and fiberwise $G$-homeomorphisms over $X$. Let $\mathcal{C}_{h}(X)$ denote the category of homotopy $G$-covering spaces and fiberwise $G$-homotopy equivalences over $X$. Let $\mathcal{C}_{w h}(X)$ denote the category of weak homotopy $G$-covering spaces and fiberwise weak $G$ equivalences over $X$.

One of the things that makes the nonequivariant theory of covering spaces useful is the existence of universal (or simply-connected) covering spaces. Equivariantly, given a $G$-space $B$, we might hope to find an equivariant covering space $p: E \rightarrow B$ each of whose restrictions to fixed sets $p^{H}: E^{H} \rightarrow B^{H}$ is a universal covering space. However, the following simple example shows that, in general, such things don't exist.

Example 1.5. Consider $G=\mathbb{Z} / 2$ acting on $S^{2}$ by reflection through the $x y$-plane, so that the $G$-fixed set is the equator. If $p: E \rightarrow S^{2}$ were a universal $G$-covering space in the sense above, then, nonequivariantly it would have to be the identity map $S^{2} \rightarrow S^{2}$, so equivariantly it would be the identity map. The restriction to the $G$-fixed set is then obviously not a universal cover.

We shall see in Section 8 that, in this example, there is a homotopy $G$-covering space $E$ such that $E \rightarrow S^{2}$ and $E^{G} \rightarrow\left(S^{2}\right)^{G}$ are both fiber-homotopy equivalent to universal covers. However, we shall also see the following example of a basespace for which there is no universal homotopy $G$-space even in this weaker sense.

Example 1.6. Consider $G=\mathbb{Z} / 2$ acting on $S^{1}$ by reflection through the $y$-axis, so that the $G$-fixed set consists of the north and south poles, $N$ and $S$. We might try to construct a universal $G$-covering space of $S^{1}$ as follows: Let $L$ be the real line with $G$ acting by multiplication by -1 and take $p: L \rightarrow S^{1}$ to be the map wrapping $L$ around the circle, with the integers going to the north pole. The map $p$ is equivariant and, nonequivariantly, is the universal covering space. The fixed-point map $p^{G}$ is the universal cover over the north pole but not the south pole, where 
$\left(p^{G}\right)^{-1}(S)=\emptyset$. Now suppose that we had a universal homotopy $G$-covering space $q: E \rightarrow S^{1}$. It would have to look like $p$ to the extent that $q^{-1}(N)$ would be a $G$ space nonequivariantly homotopy equivalent to $\mathbb{Z}$, with $G$-fixed set contractible to a single point. However, as we shall show rigorously in Section 8 , it would then have to resemble $p$ further in that $\left(q^{G}\right)^{-1}(S)$ would be empty, showing that $q$ cannot, in fact, be a universal homotopy $G$-covering space.

These examples dash any hope we may have had to to use such universal objects in, for example, defining equivariant homology and cohomology with local coefficients. Nonetheless, in Section 9 we show how to use our constructions to give a cleaner definition of homology and cohomology with local coefficients, along the lines of $[\mathbf{2}]$.

Nonequivariantly, the theory of covering spaces is intimately related to the fundamental group, or groupoid if the base space is not connected. So it is equivariantly. The following definition of the equivariant fundamental groupoid was given originally by tom Dieck $[\mathbf{1 2}, 10.7]$.

Definition 1.7. Let $X$ be a $G$-space. The equivariant fundamental groupoid $\Pi_{G} X$ of $X$ is the category whose objects are the $G$-maps $x: G / H \rightarrow X$ and whose morphisms $x \rightarrow y, y: G / K \rightarrow X$, are the pairs $(\omega, \alpha)$, where $\alpha: G / H \rightarrow G / K$ is a $G$-map and $\omega$ is an equivalence class of paths $x \rightarrow y \circ \alpha$ in $X^{H}$. As usual, two paths are equivalent if they are homotopic rel endpoints. Composition is induced by composition of maps of orbits and the usual composition of path classes.

Let $\mathcal{O}_{G}$ denote the orbit category of $G$, the category of orbits $G / H$ and $G$-maps between them. We topologize the mapping set $\mathcal{O}_{G}(G / H, G / K)$ as the manifold $(G / K)^{H}$. We have a functor $\pi: \Pi_{G} X \rightarrow \mathcal{O}_{G}$ defined by $\pi(x: G / H \rightarrow X)=G / H$ and $\pi(\omega, \alpha)=\alpha$. We topologize the mapping sets in $\Pi_{G} X$ as in $[\mathbf{1}, 3.1]$ so, in particular, $\pi$ is continuous. For each subgroup $H$, the subcategory $\pi^{-1}(G / H)$ of objects mapping to $G / H$ and morphisms mapping to the identity is a copy of $\Pi\left(X^{H}\right)$, the nonequivariant fundamental groupoid of $X^{H} \cdot \Pi_{G} X$ itself is not a groupoid in the usual sense, but a "catégories fibrées en groupoides" [6] or a "bundle of groupoids" [1] over $\mathcal{O}_{G}$.

Nonequivariantly, a covering space is determined by the action of the fundamental groupoid of the basespace on the discrete fibers. When the basespace is connected, this action is equivalent to an action of the fundamental group on the fiber over the basepoint. Our first analog in the equivariant case is given by the following definitions and Theorem A below.

\section{Definition 1.8.}

1. Let $\mathcal{D}_{G}$ be the category whose objects are the homotopy $G$-covering spaces over orbits of $G$ and whose morphisms are the $G$-fiber homotopy equivalences. That is, each object of $\mathcal{D}_{G}$ has the form $G \times_{H} F$ where $F$ is a homotopy discrete $H$-space. We give each mapping space the usual topology. Let $h \mathcal{D}_{G}$ be the homotopy category over $\mathcal{O}_{G}$ of $\mathcal{D}_{G}$.

2. Let $\mathcal{D}_{G}^{s}$ be the full subcategory of $\mathcal{D}_{G}$ consisting of the $G$-covering spaces. Note that $h \mathcal{D}_{G}^{s}=\mathcal{D}_{G}^{s}$. 
There is a map $\pi: \mathcal{D}_{G} \rightarrow \mathcal{O}_{G}$ given by taking $G \times_{H} F$ to $G / H$.

Definition 1.9. A homotopy discrete representation of $\Pi_{G} X$ is a continuous covariant functor $F: \Pi_{G} X \rightarrow h \mathcal{D}_{G}$ over $\mathcal{O}_{G}$, i.e., such that $\pi F=\pi$. A discrete representation of $\Pi_{G} X$ is a continuous covariant functor $\Pi_{G} X \rightarrow h \mathcal{D}_{G}^{s}=\mathcal{D}_{G}^{s}$ over $\mathcal{O}_{G}$. Let $\mathcal{R}_{h}(X)$ denote the category of homotopy discrete representations of $\Pi_{G} X$ and natural isomorphisms. Let $\mathcal{R}(X)$ denote the full subcategory of discrete representations of $\Pi_{G} X$.

Theorem A. Given a $G$-space $X$ of the $G$-homotopy type of a $G$-CW complex,

1. there are functors $\Delta: \mathcal{C}(X) \rightarrow \mathcal{R}(X)$ and $\nabla: \mathcal{R}(X) \rightarrow \mathcal{C}(X)$ that are inverse equivalences of categories;

2. there are functors $\Delta: \mathcal{C}_{w h}(X) \rightarrow \mathcal{R}_{h}(X)$ and $\nabla: \mathcal{R}_{h}(X) \rightarrow \mathcal{C}_{w h}(X)$ that induce inverse equivalences $\Delta: h \mathcal{C}_{w h}(X) \rightarrow \mathcal{R}_{h}(X)$ and $\nabla: \mathcal{R}_{h}(X) \rightarrow h \mathcal{C}_{w h}(X)$, where $h \mathcal{C}_{w h}(X)$ is $\mathcal{C}_{w h}(X)$ with the fiberwise weak equivalences inverted;

3. the evident functor $\mathcal{C}_{h}(X) \rightarrow \mathcal{C}_{w h}(X)$ induces an equivalence of homotopy categories.

Remark 1.10. In particular, Theorem A tells us that

1. the equivalence classes of $G$-covering spaces over $X$ are in one-to-one correspondence with the isomorphism classes of discrete representations of $\Pi_{G} X$;

2. the (weak) fiber-homotopy equivalence classes of (weak) homotopy $G$-covering spaces over $X$ are in one-to-one correspondence with the isomorphism classes of homotopy discrete representations of $\Pi_{G} X$;

3. the classification of homotopy $G$-covering spaces up to fiber homotopy and the classification of weak homotopy $G$-covering spaces up to weak fiber homotopy are equivalent problems over basespaces of the $G$-homotopy types of $G$-CW complexes. This is a general fact about fibrations, absent from [7] but noted in $[8]$.

Note that the classification given by (1) is unambiguously algebraic when $G$ is finite, in contrast to (2). To replace the remaining topological data with discrete information, we take advantage of the fact that a homotopy discrete space is determined by the system of path components of its fixed sets. This leads us to our second analog of the action of the fundamental group used in the nonequivariant case, given in the following definitions and Theorem B below.

If $F: \Pi_{G} X \rightarrow h \mathcal{D}_{G}$ is a homotopy discrete representation, there is an associated, simpler object: Let Set be the category of (discrete) sets and functions and let $\Theta F: \Pi_{G} X \rightarrow$ Set be the composite of $F$ with the contravariant functor $\Theta: \mathcal{D}_{G} \rightarrow$ Set defined as follows. If $p: E \rightarrow G / H$ is an object of $\mathcal{D}_{G}$, let

$$
\Theta(p)=\pi_{0}\left(p^{-1}(e H)^{H}\right) .
$$


Suppose given a map $[f, \sigma]: p_{1} \rightarrow p_{2}$ in $h \mathcal{D}_{G}$ as in the following diagram.

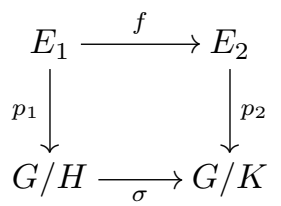

Let $\sigma(e H)=g K$. The map $\Theta[f, \sigma]$ is the composite

$$
\pi_{0}\left(p_{2}^{-1}(e K)^{K}\right) \rightarrow \pi_{0}\left(p_{2}^{-1}(g K)^{g K g^{-1}}\right) \rightarrow \pi_{0}\left(p_{2}^{-1}(g K)^{H}\right) \cong \pi_{0}\left(p_{1}^{-1}(e H)^{H}\right)
$$

where the first map is translation by $g$ while the last map is induced by $f$.

Definition 1.11. A discrete $\Pi_{G} X$-action $A$ is a continuous contravariant functor $A: \Pi_{G} X \rightarrow$ Set. (Here, continuous means locally constant and can be ignored when $G$ is finite.) We say that two $\Pi_{G} X$-actions are equivalent if they are naturally isomorphic. A strictly discrete $\Pi_{G} X$-action is one that is equivalent to $\Theta F$ for some discrete representation $F$.

Remarks 1.12 .

1. It is not obvious how to characterize the strictly discrete representations among the discrete representations in a more intrinsic way.

2. Another way of saying that a discrete $\Pi_{G} X$-action is continuous is to say that it factors through tom Dieck's "discrete fundamental groupoid" $h \Pi_{G} X$ (see $[\mathbf{1 2}, 10.9]$ and $[\mathbf{1}, 3.3])$.

Let $\mathcal{A}(X)$ denote the category of strictly discrete $\Pi_{G} X$-actions and natural isomorphisms. Let $\mathcal{A}_{h}(X)$ denote the category of discrete $\Pi_{G} X$-actions and natural isomorphisms.

Theorem B. Given a $G$-space $X$ of the G-homotopy type of a $G$-CW complex,

1. there are functors $\Phi: \mathcal{C}(X) \rightarrow \mathcal{A}(X)$ and $\Psi: \mathcal{A}(X) \rightarrow \mathcal{C}(X)$ that are inverse equivalences of categories;

2. there are functors $\Phi: \mathcal{C}_{h}(X) \rightarrow \mathcal{A}_{h}(X)$ and $\Psi: \mathcal{A}_{h}(X) \rightarrow \mathcal{C}_{h}(X)$ that induce inverse equivalences of homotopy categories.

Remark 1.13. In particular, Theorem B tells us that

1. the equivalence classes of $G$-covering spaces over $X$ are in one-to-one correspondence with the equivalence classes of strictly discrete $\Pi_{G} X$-actions; and

2. the (weak) fiber-homotopy equivalence classes of (weak) homotopy $G$-covering spaces over $X$ are in one-to-one correspondence with the equivalence classes of discrete $\Pi_{G} X$-actions.

The authors would like to thank Joseph Howard, whose undergraduate thesis was the launching point for this paper. 


\section{Some categories over $\mathcal{O}_{G}$ ( $G$ finite)}

For this section, $G$ will be finite. We shall generalize the definitions and results of this section to compact Lie groups in the following section.

In proving Theorem A, we will need to compare $\mathcal{D}_{G}$ to $h \mathcal{D}_{G}$. We would like to say that the canonical functor $\mathcal{D}_{G} \rightarrow h \mathcal{D}_{G}$ is a weak equivalence (on mapping spaces). However, it is not even continuous, in general. To get around this problem, we introduce a third category to which we can compare each of $\mathcal{D}_{G}$ and $h \mathcal{D}_{G}$. Serendipitously, this third category will also lead to an easy proof of Theorem B.

If $G / H$ is a $G$-orbit, we write $\mathcal{O}_{G \downarrow} \downarrow(G / H)$ for the category of orbits over $G / H$, i.e., the category whose objects are the $G$-maps $G / J \rightarrow G / H$ and whose maps are $G$-maps over $G / H$.

Definition 2.1. Let $\mathcal{F}_{G}$ be the category whose objects are pairs $(G / H, \alpha)$ where

$$
\alpha: \mathcal{O}_{G \downarrow}(G / H) \rightarrow \text { Set }
$$

is a contravariant functor. A map $(\sigma, f):(G / H, \alpha) \rightarrow(G / K, \beta)$ consists of a $G$-map $\sigma: G / H \rightarrow G / K$ and a natural isomorphism $f: \alpha \rightarrow \beta \circ \sigma_{*}$. We have a functor $\pi: \mathcal{F}_{G} \rightarrow \mathcal{O}_{G}$ defined by $\pi(G / H, \alpha)=G / H$ and $\pi(\sigma, f)=\sigma$.

We define a topology on the mapping sets as follows. We have an inclusion

$$
\mathcal{F}_{G}((G / H, \alpha),(G / K, \beta)) \subset \coprod_{\sigma: G / H \rightarrow G / K}\left(\prod_{\tau: G / J \rightarrow G / H} \operatorname{Set}(\alpha(\tau), \beta(\sigma \tau))\right) .
$$

We give each $\operatorname{Set}(\alpha(\tau), \beta(\sigma \tau))$ the compact-open topology and then topologize the mapping set $\mathcal{F}_{G}((G / H, \alpha),(G / K, \beta))$ as a subspace.

Note that $\mathcal{F}_{G}((G / H, \alpha),(G / K, \beta))$ is not necessarily discrete, but is totally disconnected.

We would like to define a functor $\mathcal{D}_{G} \rightarrow \mathcal{F}_{G}$, over $\mathcal{O}_{G}$, that takes a bundle $G \times_{H} F$ to the system of components of fixed-sets of $F$. In order to do the bookkeeping dealing with subgroups and their conjugates inherent in such a construction, we use the following observation: If $J \subset H$, then the space of $J$-fixed points of $F$ is homeomorphic to the space of maps over $G / H$ in the following diagram, where $\tau$ is induced by the inclusion of $J$ in $H$.

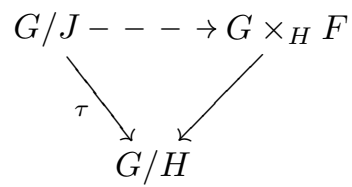

In general, if $\tau: G / J \rightarrow G / H$ is any $G$-map, there is a corresponding statement involving the fixed sets of $F$ by a conjugate of $J$. We shall write $\left(G \times_{H} F\right)^{\tau}$ for this space of maps over $G / H$. This motivates the following definition.

Definition 2.2. Define a functor $\pi_{0}: \mathcal{D}_{G} \rightarrow \mathcal{F}_{G}$ as follows. If $p: E \rightarrow G / H$ is a homotopy $G$-covering space and $\tau: G / J \rightarrow G / H$, let

$$
\pi_{0}(p)(\tau)=\pi_{0}\left(E^{\tau}\right),
$$


the set of components of $E^{\tau}$. It is easy to see that this defines a contravariant functor of $\tau$ and a covariant functor of $p$.

If we ignore topologies, it is easy to see that $\pi_{0}$ factors through $h \mathcal{D}_{G}$ to give a functor $h \mathcal{D}_{G} \rightarrow \mathcal{F}_{G}$ we call $\pi_{0}$ as well.

Lemma 2.3. The functors $\pi_{0}: \mathcal{D}_{G} \rightarrow \mathcal{F}_{G}$ and $\pi_{0}: h \mathcal{D}_{G} \rightarrow \mathcal{F}_{G}$ are both continuous and weak equivalences on mapping spaces. There is a continuous functor $\psi: \mathcal{F}_{G} \rightarrow$ $\mathcal{D}_{G}$ such that the induced (not necessarily continuous) functor $\mathcal{F}_{G} \rightarrow h \mathcal{D}_{G}$ is an inverse equivalence to $\pi_{0}: h \mathcal{D}_{G} \rightarrow \mathcal{F}_{G}$.

Proof. We define $\psi: \mathcal{F}_{G} \rightarrow \mathcal{D}_{G}$ as follows. If $(G / H, \alpha)$ is an object of $\mathcal{F}_{G}$, let

$$
\psi(G / H, \alpha)=C \alpha=B\left(\alpha, \mathcal{O}_{G \downarrow} \downarrow(G / H), J\right),
$$

a generalization of Elmendorf's construction in [4] (a similar construction was used in $[\mathbf{2}, \S 4])$. Here, $J$ is the functor from $\mathcal{O}_{G \downarrow} \downarrow(G / H)$ to the category of $G$-spaces over $G / H$ that takes an orbit over $G / H$ to itself, and $B$ is the two-sided bar construction as in [4]. The result, $C \alpha$, is a $G$-space over $G / H$ with the property that $(C \alpha)^{\tau}$ is homotopy equivalent to $\alpha(\tau)$ for every $\tau$. As a result, $\pi_{0} \circ \psi$ is naturally isomorphic to the identity.

If we let $h \psi: \mathcal{F}_{G} \rightarrow h \mathcal{D}_{G}$ be the induced functor, then, using the fact that the fibers of objects in $h \mathcal{D}_{G}$ are homotopy discrete, it follows as in [4] that $h \psi \circ \pi_{0}$ is also naturally isomorphic to the identity.

That $\pi_{0}: h \mathcal{D}_{G} \rightarrow \mathcal{F}_{G}$ is continuous follows trivially because the source is discrete. Since $\pi_{0}$ is an equivalence of categories, it is bijective on each morphism space. However, a bijection from a discrete space to a totally disconnected space, as we have here, is a weak equivalence.

We now turn to $\pi_{0}: \mathcal{D}_{G} \rightarrow \mathcal{F}_{G}$, and first show that it is continuous on morphism spaces. So, let $p_{1}: E_{1} \rightarrow G / H$ and $p_{2}: E_{2} \rightarrow G / K$ be two objects of $\mathcal{D}_{G}$. Let $f: E_{1} \rightarrow E_{2}$ be a $G$-map over $\sigma: G / H \rightarrow G / K$. Let $\pi_{0}(f)=(\sigma, \bar{f})$, so $\bar{f}: \pi_{0}\left(p_{1}\right) \rightarrow$ $\pi_{0}\left(p_{2}\right) \circ \sigma_{*}$. For a typical subbasic open neighborhood of $(\sigma, \bar{f})$, we can take

$$
V=\{(\sigma, \bar{g}) \mid \bar{f}(\tau)(\bar{x})=\bar{g}(\tau)(\bar{x})\}
$$

for a chosen $\tau: G / J \rightarrow G / H$ and $\bar{x} \in \pi_{0}\left(p_{1}\right)(\tau)$. The inverse image $\pi_{0}^{-1}(V)$ is then open, since

$$
\pi_{0}^{-1}(V)=\{g \mid f(x) \text { is path-connected to } g(x)\}
$$

where $x$ is a chosen point in the component of $\left(E_{1}\right)^{\tau}$ corresponding to $\bar{x}$. (Precisely, a point in $\left(E_{1}\right)^{\tau}$ is a map $G / J \rightarrow E_{1}$ and we take $x$ to be the image of $\left.e J.\right)$

Now we already know that $\pi_{0}$ induces a bijection on the set of components of each morphism space. To show that $\pi_{0}$ is a weak equivalence on morphism spaces, it suffices, then, to show that the higher homotopy groups vanish for the morphism spaces in $\mathcal{D}_{G}$. Since the objects of $\mathcal{D}_{G}$ have the form $G \times_{H} F$, where $F$ is homotopy discrete, the claim follows from elementary equivariant obstruction theory. 


\section{Some categories over $\mathcal{O}_{G}(G$ compact Lie $)$}

We now discuss the generalization of the definitions and results of the preceding section to compact Lie groups. In constructing $\mathcal{F}_{G}$ we need to take into account the topology on both the set of morphisms and the set of objects of $\mathcal{O}_{G} \downarrow(G / H)$. Thus, we consider $\mathcal{O}_{G \downarrow}(G / H)$ to be the topological category (= category internal to the category of topological spaces) with object space

$$
O=\operatorname{Ob}\left(\mathcal{O}_{G \downarrow} \downarrow(G / H)\right)=\coprod_{G / J} \mathcal{O}_{G}(G / J, G / H)
$$

and morphism space

$$
M=\operatorname{Map}\left(\mathcal{O}_{G} \downarrow(G / H)\right)=\coprod_{G / J, G / K} \mathcal{O}_{G}(G / J, G / H) \times \mathcal{O}_{G}(G / K, G / J) .
$$

The source and target maps $S, T: M \rightarrow O$ are given by $S(\sigma, \tau)=\sigma \circ \tau$ and $T(\sigma, \tau)=$ $\sigma$. The identity and composition maps are the obvious ones.

In place of contravariant functors on $\mathcal{O}_{G} \downarrow(G / H)$ we will use right $\mathcal{O}_{G} \downarrow(G / H)$ modules, defined as follows. (These are called "actions" in [5], but we have already used that term for another concept.)

Definition 3.1. Let $\mathcal{C}$ be a topological category, with object space $O$ and morphism space $M$. A right $\mathcal{C}$-module is a space $A$ together with a continuous map $\alpha: A \rightarrow O$ and a continuous map $\mu: A \times_{O} M \rightarrow A$ over $O$. Here, $A \times_{O} M$ denotes the pullback in the following diagram:

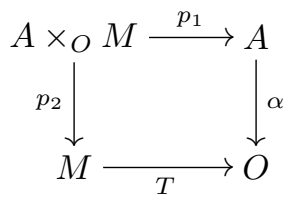

To say that $\mu$ is a map over $O$ is to say that the following diagram commutes:

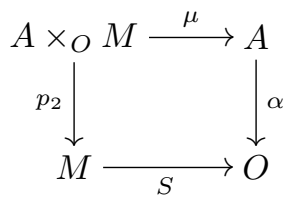

We require that $\mu$ be unital and associative in the obvious way.

We shall sometimes abbreviate $(A, \alpha, \mu)$ by $\alpha$. We think of $\alpha: A \rightarrow O$ as specifying a "continuously varying" functor on $\mathcal{C}$, whose value at an object $c \in O$ is $\alpha^{-1}(c)$. In particular, the following modules take the place of set-valued functors.

Definition 3.2. A discrete right $\mathcal{C}$-module $(A, \alpha, \mu)$ is one in which $\alpha^{-1}(c)$ is a discrete space for every object $c \in O$.

In the case $\mathcal{C}=\mathcal{O}_{G} \downarrow(G / H)$, these modules have a very nice form.

Proposition 3.3. If $(A, \alpha, \mu)$ is a right $\mathcal{O}_{G} \downarrow(G / H)$-module, then $\alpha$ is a fiber bundle (whose fibers may vary over different components). 
Proof. Let $O=\operatorname{Ob}\left(\mathcal{O}_{G} \downarrow(G / H)\right)$ and let $M=\operatorname{Map}\left(\mathcal{O}_{G} \downarrow(G / H)\right)$. Let $\sigma: G / J \rightarrow$ $G / H$ be an object in $O$ and let $U$ be a contractible open neighborhood of $\sigma$ in $\mathcal{O}_{G}(G / J, G / H)$. We show that $\alpha^{-1}(U)$ is homeomorphic to $\alpha^{-1}(\sigma) \times U$ over $U$.

The map $\sigma_{*}: \mathcal{O}_{G}(G / J, G / J) \rightarrow \mathcal{O}_{G}(G / J, G / H)$ is the fiber bundle $(G / J)^{J} \rightarrow$ $(G / H)^{J}$. Let $\tilde{s}: U \rightarrow \mathcal{O}_{G}(G / J, G / J)$ be a section with $\tilde{s}(\sigma)=1_{G / J}$. Write $s$ for the map $U \rightarrow \mathcal{O}_{G}(G / J, G / H) \times \mathcal{O}_{G}(G / J, G / J) \subset M$ defined by $s(\tau)=(\sigma, \tilde{s}(\tau))$; we have $S(s(\tau))=\tau$ and $T(s(\tau))=\sigma$ for all $\tau \in U$.

Since $\mathcal{O}_{G}(G / J, G / J)=N J / J$, we have $\tilde{s}^{-1}: U \rightarrow \mathcal{O}_{G}(G / J, G / J)$, the pointwise inverse of $\tilde{s}$. Let $s^{-1}(\tau)=\left(\tau, \tilde{s}^{-1}(\tau)\right)$. Then $S\left(s^{-1}(\tau)\right)=\sigma$ and $T\left(s^{-1}(\tau)\right)=\tau$.

We can now define $\phi: \alpha^{-1}(\sigma) \times U \rightarrow \alpha^{-1}(U)$ over $U$ by $\phi(a, \tau)=\mu(a, s(\tau))$. We define $\psi: \alpha^{-1}(U) \rightarrow \alpha^{-1}(\sigma) \times U$ by $\psi(b)=\left(\mu\left(b, s^{-1}(\alpha(b))\right), \alpha(b)\right)$. It is straightforward to check, using the associativity of $\mu$, that $\phi$ and $\psi$ are inverse homeomorphisms.

In particular, the special case we are interested in is this:

Corollary 3.4. If $(A, \alpha, \mu)$ is a discrete right $\mathcal{O}_{G \downarrow} \downarrow(G / H)$-module, then $\alpha$ is a covering map.

We now redefine $\mathcal{F}_{G}$ to include the case when $G$ is a compact Lie group.

Definition 3.5. Let $\mathcal{F}_{G}$ be the category whose objects are pairs $(G / H,(A, \alpha, \mu))$ where $(A, \alpha, \mu)$ is a discrete right $\mathcal{O}_{G} \downarrow(G / H)$-module. A map from $(G / H,(A, \alpha, \mu))$ to $(G / K,(B, \beta, \nu))$ is a pair $(\sigma, f)$ where $\sigma: G / H \rightarrow G / K$ is a $G$-map and $f: A \rightarrow B$ is a map over $\sigma_{*}$ that respects the module actions and is a one-to-one correspondence on each fiber of $\alpha$. The functor $\pi: \mathcal{F}_{G} \rightarrow \mathcal{O}_{G}$ is defined as in 2.1. We topologize the set of maps from $(G / H,(A, \alpha, \mu))$ to $(G / K,(B, \beta, \nu))$ as a subspace of $\mathcal{O}_{G}(G / H, G / K) \times \operatorname{map}(A, B)$.

Since maps between covering spaces are determined by their restrictions to a single fiber over each component, it follows that each fiber of $\pi: \mathcal{F}_{G}((G / H, \alpha)$, $(G / K, \beta)) \rightarrow \mathcal{O}_{G}(G / H, G / K)$ is totally disconnected. Moreover, we have the following result.

Proposition 3.6. The map $\pi: \mathcal{F}_{G}((G / H, \alpha),(G / K, \beta)) \rightarrow \mathcal{O}_{G}(G / H, G / K)$ is a fibration.

Proof. Let $\alpha=(A, \alpha, \mu)$ and $\beta=(B, \beta, \nu)$. Consider a lifting problem as in the following diagram.

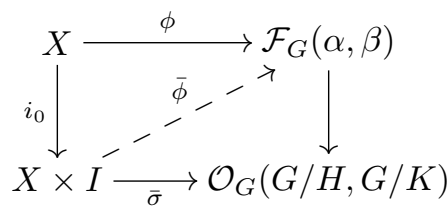

For brevity, write

$$
O_{G / H}=\mathrm{Ob}_{G} \downarrow(G / H)
$$


and

$$
M_{G / H}=\operatorname{Map} \mathcal{O}_{G \downarrow}(G / H)
$$

and similarly for $G / K$. The map $\phi$ in the diagram above is equivalent to a pair of maps, $\sigma: X \times G / H \rightarrow G / K$ and $f: X \times A \rightarrow B$ making the following diagram commute.

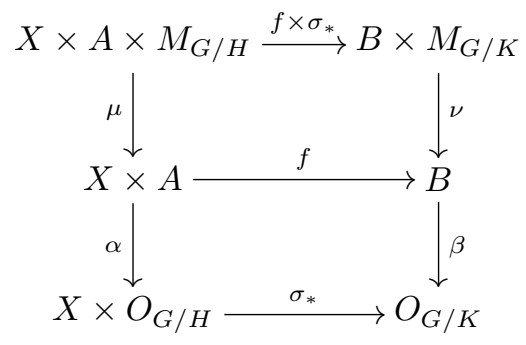

We are already given an extension of $\sigma$ to $\bar{\sigma}: X \times G / H \times I \rightarrow G / K$. We need to extend $f$ to $\bar{f}: X \times A \times I \rightarrow B$ making the following diagram commute.

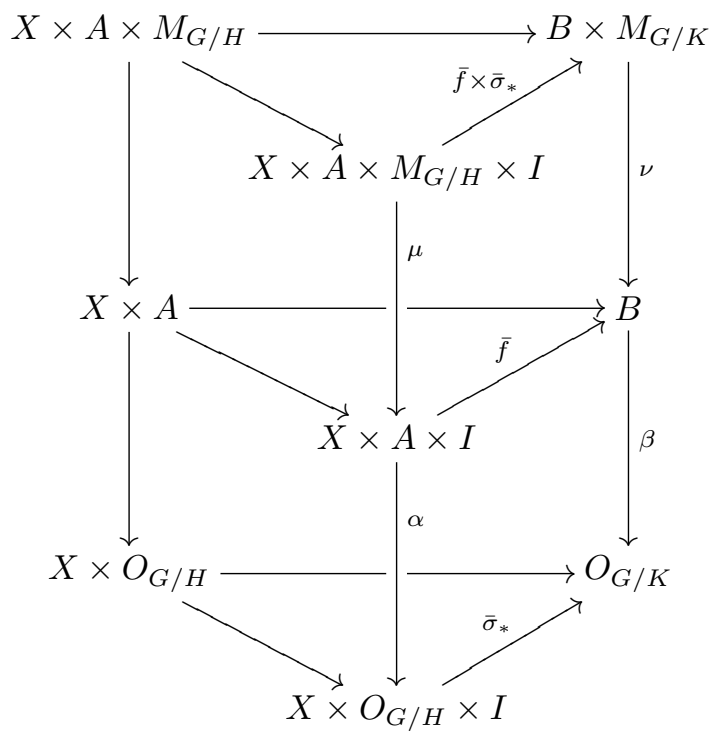

Because $\beta$ is a covering map, there is a unique $\bar{f}$ making the bottom half of the diagram commute. Also, the larger rectangle commutes:

$$
\beta \circ \bar{f} \circ \mu=\bar{\sigma}_{*} \circ \alpha \circ \mu=\beta \circ \nu \circ\left(\bar{f} \times \bar{\sigma}_{*}\right),
$$

so $\bar{f} \circ \mu$ and $\nu \circ\left(\bar{f} \times \bar{\sigma}_{*}\right)$ can be thought of as two lifts of the same homotopy, starting at the same initial point. Using again the fact that $\beta$ is a covering map, $\bar{f} \circ \mu=\nu \circ\left(\bar{f} \times \bar{\sigma}_{*}\right)$ and the whole diagram commutes. This gives us the lift $\bar{\phi}$ in our original diagram.

Definition 3.7. Define a continuous covariant functor $\pi_{0}: \mathcal{D}_{G} \rightarrow \mathcal{F}_{G}$ as follows. If 
$p: E \rightarrow G / H$ is a homotopy $G$-covering space, let

$$
\pi_{0}(p)=\left(\coprod_{G / J} \pi_{0}^{\mathrm{f}} \operatorname{map}_{G}(G / J, E), p_{*}, \mu\right) .
$$

Here, $\pi_{0}^{\mathrm{f}}$ denotes the fiberwise discretization (given the quotient topology) of the bundle

$$
\operatorname{map}_{G}(G / J, E) \rightarrow \operatorname{map}_{G}(G / J, G / H),
$$

and $p_{*}$ and $\mu$ are the obvious maps given by composition. The value of $\pi_{0}$ on a morphism $f$ is given by composition with $f$.

We can now generalize Lemma 2.3 to compact Lie groups.

Lemma 3.8. The functors $\pi_{0}: \mathcal{D}_{G} \rightarrow \mathcal{F}_{G}$ and $\pi_{0}: h \mathcal{D}_{G} \rightarrow \mathcal{F}_{G}$ are both continuous and weak equivalences on mapping spaces. There is a continuous functor $\psi: \mathcal{F}_{G} \rightarrow$ $\mathcal{D}_{G}$ such that the induced (not necessarily continuous) functor $\mathcal{F}_{G} \rightarrow h \mathcal{D}_{G}$ is an inverse equivalence to $\pi_{0}: h \mathcal{D}_{G} \rightarrow \mathcal{F}_{G}$.

Proof. We define $\psi: \mathcal{F}_{G} \rightarrow \mathcal{D}_{G}$ as follows. If $(G / H, \alpha)$ is an object of $\mathcal{F}_{G}$, let

$$
\psi(G / H, \alpha)=B\left(\alpha, \mathcal{O}_{G \downarrow}(G / H), J\right) .
$$

Here, $J$ is the left $\mathcal{O}_{G \downarrow}(G / H)$-module given by the projection

$$
J: \bar{J}=\coprod_{G / L} \mathcal{O}_{G}(G / L, G / H) \times G / L \rightarrow \coprod_{G / L} \mathcal{O}_{G}(G / L, G / H),
$$

and comes with a map $\bar{J} \rightarrow G / H$ given by evaluation. $B\left(\alpha, \mathcal{O}_{G \downarrow}(G / H), J\right)$ is the geometric realization of the simplicial $G$-space whose $n$th space is $A \times_{O} M_{G / H}^{n} \times{ }_{O} \bar{J}$ $\left(M_{G / H}^{n}\right.$ denotes the $n$-fold product over $\left.O\right) ; B\left(\alpha, \mathcal{O}_{G \downarrow}(G / H), J\right)$ is a space over $G / H$ via the map from $\bar{J}$ to $G / H$. By the general properties of the bar construction, this gives a homotopy covering space over $G / H$ such that $\pi_{0} \psi(G / H, \alpha)$ is naturally isomorphic to $\alpha$. It follows, as in 2.3 , that $h \psi \circ \pi_{0}$ is also naturally isomorphic to the identity.

To show that $\pi_{0}$ is continuous, we need to show that, if $(\sigma, f)$ varies continuously in this diagram:

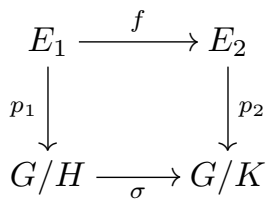

then $\left(\sigma_{*}, f_{*}\right)$ varies continuously in this diagram:

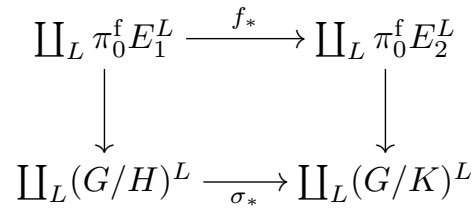


We can reduce to the case of an open neighborhood of $\left(\sigma_{*}, f_{*}\right)$ consisting of those $\left(\sigma_{*}^{\prime}, g\right)$ such that $\sigma^{\prime}$ is in some contractible neighborhood of $\sigma$ and $f_{*}$ and $g$ are homotopic on a compact set, over a homotopy of $\sigma$ and $\sigma^{\prime}$ in the given contractible neighborhood. As in the finite case, the preimage of this open set can be checked to be open in $\mathcal{D}_{G}\left(p_{1}, p_{2}\right)$.

The remainder of the proof can be adopted fiberwise from the proof of Lemma 2.3, the global conclusions following from the fact that both $\mathcal{D}_{G}\left(p_{1}, p_{2}\right) \rightarrow$ $\mathcal{O}_{G}(G / H, G / K)$ and $\mathcal{F}_{G}\left(\pi_{0}\left(p_{1}\right), \pi_{0}\left(p_{2}\right)\right) \rightarrow \mathcal{O}_{G}(G / H, G / K)$ are fibrations.

\section{Some results on fibrations}

Our (homotopy) covering spaces are fibrations whose fibers are, in general, not homotopy equivalent to compact spaces. We need the following two results pertaining to fiberwise approximation by $\mathrm{CW}$ complexes, which strengthen the classification of fibrations and are due in the nonequivariant case to May [8].

Theorem 4.1. If $X$ has the $G$-homotopy type of a $G$-CW complex and $p: E \rightarrow X$ is a $G$-fibration, then $p$ is fiberwise weakly equivalent to a $G$-fibration whose fibers have the equivariant homotopy type of equivariant $C W$ complexes.

Proof. The following is based on the argument in [8, 1.1]. Let $C p: C E \rightarrow C X$ be a $G$-CW approximation of $p$ (see, for example, $[\mathbf{1 3}, 3.7]$ ), giving us the following commutative diagram. (If your favorite construction gives a diagram that commutes only up to homotopy, we can use the fact that $p$ is a fibration to make it commute on the nose.)

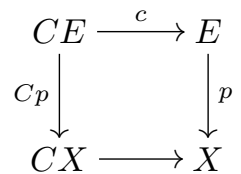

The top horizontal arrow, $c$, is a weak $G$-equivalence while the bottom one is a $G$-homotopy equivalence. Now, $C p$ may not be a fibration so let $\Gamma C p: \Gamma C E \rightarrow C X$ be the associated path fibration. We then have the following commutative diagram.

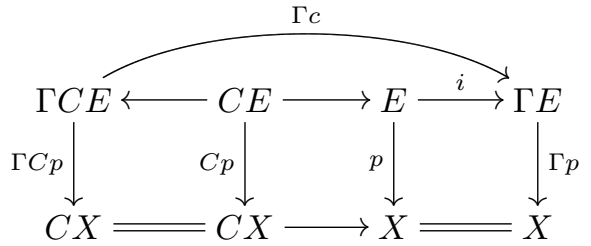

Since $p$ is a fibration, $i$ is a fiberwise homotopy equivalence. Let $j: \Gamma E \rightarrow E$ be a fiberwise homotopy inverse. The composite $j \circ \Gamma c$ is then a fiberwise map and an equivariant fiberwise weak equivalence by the 5-lemma. Further, since $\Gamma C E$ is $G$-homotopy equivalent to $C E$, it has the $G$-homotopy type of a $G$-CW complex. It follows from $[\mathbf{1 3}, 4.14]$ that the fibers of $\Gamma C p$ have the equivariant homotopy types of CW complexes. 
Finally, since $X$ has the $G$-homotopy type of a $G$-CW complex, $c: C X \rightarrow X$ is a $G$-homotopy equivalence. Let $d: X \rightarrow C X$ be a $G$-homotopy inverse and consider the following diagram, in which $E^{\prime}$ is the pullback in the left square.

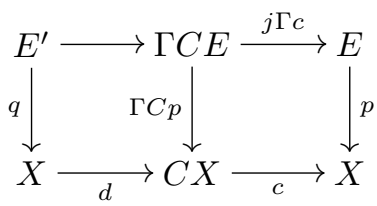

Being the pullback of $\Gamma C p, q$ is a fibration whose fibers have the equivariant homotopy types of CW complexes. Moreover, since the composite $c \circ d$ is $G$-homotopic to the identity, we can homotope the map $E^{\prime} \rightarrow E$ to a map over $X$, exhibiting the required fiberwise weak equivalence.

Theorem 4.2. If $X$ has the $G$-homotopy type of a $G$-CW complex and if $p_{1}: E_{1} \rightarrow$ $X$ and $p_{2}: E_{2} \rightarrow X$ are $G$-fibrations whose fibers have the equivariant homotopy type of equivariant $C W$ complexes, then $p_{1}$ and $p_{2}$ are $G$-fiber homotopy equivalent if and only if they are weakly fiber homotopy equivalent.

Proof. Suppose that $p_{1}$ and $p_{2}$ have the same weak fiberwise homotopy type. Then they are connected by a sequence of fiberwise weak equivalences (some going the "wrong way"), but the fibers of the intermediate fibrations need not have the homotopy types of CW complexes. However, this problem is solved by replacing each intermediate fibration with one whose fibers do have the homotopy types of CW complexes, using the preceding theorem. Moreover, the total space of each of these fibrations has the $G$-homotopy type of a $G$-CW complex, by $[\mathbf{1 4}, 6.1]$, the equivariant version of $[\mathbf{1 1}]$ (as proved in $[\mathbf{1 0}]$ ). (Note that the countability assumption in $[\mathbf{1 4}, 6.1]$ is unnecessary.) We can therefore replace all fiberwise weak equivalences with fiberwise homotopy equivalences.

\section{Proof of Theorem A}

For the sake of simplicity, we shall work first with weak homotopy $G$-covering spaces, and address homotopy $G$-covering spaces at the end of the section.

We begin by defining $\Delta: \mathcal{C}_{w h}(X) \rightarrow \mathcal{R}_{h}(X)$. Let $p: E \rightarrow X$ be a weak homotopy $G$-covering space. On an object $x: G / H \rightarrow X, \Delta(p)$ is given by

$$
\Delta(p)(x)=x^{*}(p)
$$

the pullback bundle. $\Delta(p)$ is given on morphisms using the $G$-covering homotopy property.

To define $\nabla$ we first need the following definition, which is Definition 24.3 of $[\mathbf{1}]$.

Definition 5.1. The Moore path category $\Lambda_{G} X$ is the topological category whose objects are those of $\Pi_{G} X$ and whose morphisms from $x: G / H \rightarrow X$ to $y: G / K \rightarrow Y$ are the triples $(\lambda, r, \alpha)$, where $\alpha: G / H \rightarrow G / K$ is a $G$-map, $r \geqslant 0$ is a real number, and $\lambda: G / H \times[0, r] \rightarrow X$ is a path of length $r$ from $x$ to $y \circ \alpha$ in $X^{H}$. Composition is given by concatenation of paths and addition of real numbers. Regarding paths 
as defined on all of $[0, \infty]$ by letting them be eventually constant, the set of paths from $x$ to $y$ is topologized as a subspace of the product of $[0, \infty)$ and the space of maps $[0, \infty] \rightarrow X^{H}$. Let $\pi: \Lambda_{G} X \rightarrow \mathcal{O}_{G}$ be the functor given by $\pi(x)=G / H$ and $\pi(\lambda, r, \alpha)=\alpha$.

There is an evident functor $\omega: \Lambda_{G} X \rightarrow \Pi_{G} X$ shown to be continuous in [1].

Now we define $\nabla: \mathcal{R}_{h}(X) \rightarrow \mathcal{C}_{w h}(X)$ as follows. Let $F: \Pi_{G} X \rightarrow h \mathcal{D}_{G}$ be a representation. Let

$$
\bar{F}=\psi \circ \pi_{0} \circ F \circ \omega: \Lambda_{G} X \rightarrow \Pi_{G} X \rightarrow h \mathcal{D}_{G} \rightarrow \mathcal{F}_{G} \rightarrow \mathcal{D}_{G} .
$$

By the argument of $[\mathbf{7}, 7.6]$, the map $q: B\left(P_{G} X, \Lambda_{G} X, \bar{F}\right) \rightarrow B\left(P_{G} X, \Lambda_{G} X, J\right)$ is a $G$-quasifibration, where $B$ denotes the two-sided bar construction, as in the proof of Lemma 2.3, $P_{G} X$ is the contravariant Moore path space functor used in [1, 24.5], and $J(x: G / H \rightarrow X)=G / H$. By $[\mathbf{1}, 24.5]$, the map $\epsilon: B\left(P_{G} X, \Lambda_{G} X, J\right) \rightarrow X$ is a weak $G$-equivalence; we are assuming that $X$ is $G$-CW, hence there is an inverse weak $G$-equivalence $\zeta: X \rightarrow B\left(P_{G} X, \Lambda_{G} X, J\right)$. Let $\nabla(F)$ be the pullback to $X$ along $\zeta$ of the $G$-fibration associated to $q$.

We first show that $\Delta \nabla(F)$ is naturally equivalent to $F$ for any representation $F$. Since $\epsilon$ and $\zeta$ are inverse weak $G$-equivalences, they induce inverse equivalences $\epsilon_{*}$ and $\zeta_{*}$ of fundamental groupoids. Let $\eta: \Pi_{G} X \rightarrow \Pi_{G} B\left(P_{G} X, \Lambda_{G} X, J\right)$ be given on objects by the inclusion of vertices, extended to maps so that $\epsilon_{*} \circ \eta$ is the identity. It follows that there is a natural isomorphism $\zeta_{*} \rightarrow \eta$. It is easy to see that $\Delta \nabla(F)$ is naturally isomorphic to the pullback along $\zeta_{*}$ of the representation of $\Pi_{G} B\left(P_{G} X, \Lambda_{G} X, J\right)$ induced by the fibration associated to $q$. On the other hand, the pullback along $\eta$ of that same representation is clearly equivalent to the original $F$. Therefore, $\Delta \nabla(F)$ is equivalent to $F$.

That $\nabla \Delta(p)$ is naturally equivalent to $p$ for a weak homotopy covering space $p: E \rightarrow X$ follows from the following diagram of equivalences of $G$-quasifibrations.

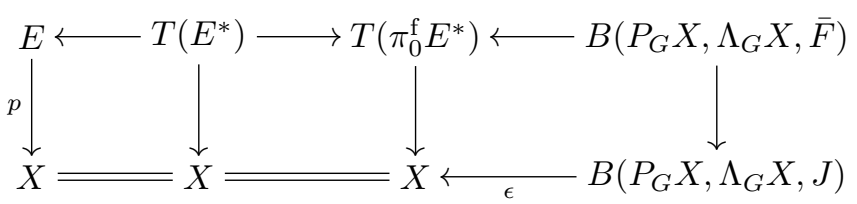

Here, $T$ is Elemendorf's original construction: If $Y^{*}$ is a contravariant functor on $\mathcal{O}_{G}$ then $T\left(Y^{*}\right)=B\left(Y^{*}, \mathcal{O}_{G}, J\right) . E^{*}$ is the contravariant functor on $\mathcal{O}_{G}$ with $E^{*}(G / H)=E^{H}$, and $\pi_{0}^{\mathrm{f}} E^{*}$ is the similar functor in which we replace each fiber in $E^{H} \rightarrow X^{H}$ with its set of components (and give the resulting set the quotient topology). It is straightforward to check that each vertical map is a $G$-quasifibration, that each horizontal map on the top line is a fiberwise equivariant equivalence, and the bottom maps are equivariant equivalences. Since the map $\zeta: X \rightarrow B\left(P_{G} X, \Lambda_{G} X, J\right)$ is obtained by inverting $\epsilon$, the diagram shows that $p$ is equivalent to $\nabla \Delta(p)$ as claimed. Thus we've shown part (2) of Theorem A.

Part (3) follows from Theorems 4.1 and 4.2.

Finally, part (1) follows from part (3) and the following observations. If $p$ is a $G$-covering space, then $\Delta(p)$ is a discrete representation. On the other hand, if $p$ is 
a homotopy $G$-covering space and $\Delta(p)$ is discrete, then we can take the fiberwise discretization of $p$, meaning that we replace each fiber with its set of components and give the result the quotient topology. Given our topological assumptions on the fibers and the base space, the result is a $G$-covering space weakly fiberwise $G$-equivalent to $p$.

\section{Proof of Theorem B}

Let $\mathcal{B}_{h}(X)$ be the category whose objects are the functors $\Pi_{G} X \rightarrow \mathcal{F}_{G}$ over $\mathcal{O}_{G}$ and whose morphisms are the natural isomorphisms. By Lemmas 2.3 and 3.8 we have the functor

$$
\left(\pi_{0}\right)_{*}: \mathcal{R}_{h}(X) \rightarrow \mathcal{B}_{h}(X)
$$

and $\left(\pi_{0}\right)_{*}$ induces an equivalence of homotopy categories.

Now define

$$
\phi: \mathcal{B}_{h}(X) \rightarrow \mathcal{A}_{h}(X)
$$

as follows. If $F: \Pi_{G} X \rightarrow \mathcal{F}_{G}$ is a functor over $\mathcal{O}_{G}$ and $x: G / H \rightarrow X$ is an object of $\Pi_{G} X$, let

$$
\phi(F)(x)=F(x)\left(1_{G / H}\right) .
$$

(We describe the construction as if $F(x)$ were a functor, as in Section 2. It is straightforward to translate to the language of modules used in Section 3.) If $y: G / K \rightarrow X$, then on a morphism $(\omega, \alpha): x \rightarrow y, \phi(F)(\omega, \alpha)$ is the composite

$$
F(y)\left(1_{G / K}\right) \stackrel{\alpha^{*}}{\longrightarrow} F(y)(\alpha) \stackrel{F(\omega, \alpha)\left(1_{G / H}\right)^{-1}}{\longrightarrow} F(x)\left(1_{G / H}\right) .
$$

Here, the first arrow is induced by the map $\alpha: \alpha \rightarrow 1_{G / K}$ in $\mathcal{O}_{G} \downarrow(G / K)$. (Recall that $\alpha: G / H \rightarrow G / K$.) It is straightforward to check that $\phi(F)$ is a contravariant functor and that $\phi$ is natural in $F$.

The inverse,

$$
\psi: \mathcal{A}_{h}(X) \rightarrow \mathcal{B}_{h}(X)
$$

is defined as follows when $G$ is finite. If $A: \Pi_{G} X \rightarrow$ Set is a contravariant functor, $x: G / H \rightarrow X$ is an object of $\Pi_{G} X$, and $\alpha: G / J \rightarrow G / H$, let

$$
\psi(A)(x)(\alpha)=A(x \circ \alpha) .
$$

If $\beta: G / K \rightarrow G / H$ and $\gamma: \alpha \rightarrow \beta$ is a map in $\mathcal{O}_{G \downarrow} \downarrow(G / H)$, so $\alpha=\beta \circ \gamma$, let

$$
\psi(A)(x)(\gamma)=A(1, \gamma): A(x \circ \beta) \rightarrow A(x \circ \alpha) .
$$

One checks that $\psi(A)(x)$ is a contravariant functor on $\mathcal{O}_{G} \downarrow(G / H)$, hence an object of $\mathcal{F}_{G}$ over $G / H$. For the covariance in $x$, let $y: G / K \rightarrow X$ and let $(\omega, \alpha): x \rightarrow y$ be a map in $\Pi_{G} X$. We then define $\psi(A)(\omega, \alpha)$ to be the natural isomorphism from $\psi(A)(x)$ to $\psi(A)(y) \circ \alpha_{*}$ given on an object $\beta: G / J \rightarrow G / H$ by

$$
\psi(A)(\omega, \alpha)(\beta)=A(\omega, 1): A(x \circ \beta) \rightarrow A(y \circ \alpha \circ \beta) .
$$

It is straightforward to check that this makes $\psi(A)$ a covariant functor on $\Pi_{G} X$, natural in $A$. 
Another straightforward check shows that $\phi$ and $\psi$ are inverse equivalences of categories. The composite $\phi \circ\left(\pi_{0}\right)_{*}$ is, therefore, an equivalence of homotopy categories. Combining this with Theorem A gives part (2) of Theorem B when $G$ is finite.

When $G$ is infinite we have to take some care to define the topology necessary to make $\psi(A)(x)$ a right $\mathcal{O}_{G} \downarrow(G / H)$-module. Consider the induced functor

$$
x_{*}: \Pi_{G}(G / H) \rightarrow \Pi_{G} X .
$$

If $\pi: \Pi_{G}(G / H) \rightarrow \mathcal{O}_{G}$ is the usual functor, we have $\pi^{-1}(G / J)=\Pi \mathcal{O}_{G}(G / J, G / H)$, the nonequivariant fundamental groupoid. Composing with $A$ and restricting to these fibers gives a functor

$$
A x_{*}: \coprod_{G / J} \Pi \mathcal{O}_{G}(G / J, G / H) \rightarrow \text { Set. }
$$

By the nonequivariant theory of covering spaces, this defines a covering space $E_{x} \rightarrow$ $O_{G / H}$ whose fiber over a map $\alpha: G / J \rightarrow G / H$ is $A(x \circ \alpha)$. One can check that the action of morphisms is continuous, making $\psi(A)(x)$ a right $\mathcal{O}_{G} \downarrow(G / H)$-module. The rest of the argument follows as in the finite case.

Part (1) of Theorem B follows from the definition of $\mathcal{A}(X)$.

\section{Classifying spaces}

In the preceding section we completed the algebraic classification of (homotopy) covering spaces. In this section we relate these results to the homotopy classification via classifying spaces.

Now, the collection of homotopy classes of maps from a space into a classifying space is a set, whereas the collections of equivalence classes of (weak homotopy) $G$-covering spaces and of (homotopy) discrete representations of $\Pi_{G} X$ are proper classes, not sets. However, if we restrict the cardinality of the fibers these collections become sets. So, assume that $\kappa$ is some cardinal number.

Definition 7.1. Let $X$ be a $G$-space.

1. Let $\mathcal{C}(X)$ and $\mathcal{C}_{h}(X)$ be the categories defined in 1.4. Let $\tilde{\mathcal{C}}(X)$ and $\tilde{\mathcal{C}}_{h}(X)$ be the full subcategories given by the (homotopy) $G$-covering spaces over $X$ in which the cardinality of the set of components of each fixed set of each fiber is less than $\kappa$. Let $\mathcal{E} \tilde{\mathcal{C}}(X)$ and $\mathcal{E} \tilde{\mathcal{C}}_{h}(X)$ be the collections of homotopy equivalence classes of objects in these categories. It follows from Theorem B that these collections are sets.

2. Let $\mathcal{R}(X)$ and $\mathcal{R}_{h}(X)$ be the categories defined in 1.9. Let $\tilde{\mathcal{R}}(X)$ and $\tilde{\mathcal{R}}_{h}(X)$ be the full subcategories given by the (homotopy) discrete representations of $\Pi_{G} X$ in which the cardinality of the set of components of each fixed set of each fiber is less than $\kappa$. Let $\mathcal{E} \tilde{\mathcal{R}}(X)$ and $\mathcal{E} \tilde{\mathcal{R}}_{h}(X)$ be the collections of equivalence classes of objects in these categories. It follows from Theorems A and B that these collections are sets.

3. Let $\mathcal{D}_{G}$ and $h \mathcal{D}_{G}$ be the categories defined in 1.8. Let $h \tilde{\mathcal{D}}_{G}$ be any small subcategory equivalent over $\mathcal{O}_{G}$ to the full subcategory of $h \mathcal{D}_{G}$ consisting of 
those $G$-covering spaces in which the cardinality of the set of components of each fixed set of each fiber is less than $\kappa$. Let $\tilde{\mathcal{D}}_{G}$ be the full subcategory of $\mathcal{D}_{G}$ on the set of objects of $h \tilde{\mathcal{D}}_{G}$. Let $\tilde{\mathcal{D}}_{G}^{s}$ be defined similarly.

4. Let $\mathcal{F}_{G}$ be the category defined in 2.1. Let $\tilde{\mathcal{F}}_{G}$ be formed from $\mathcal{F}_{G}$ in a manner similar to the way the categories above were formed, chosen to contain the objects in the image of $\pi_{0}$ restricted to $\tilde{\mathcal{D}}_{G}$.

The fact that $h \tilde{\mathcal{D}}_{G}$ and $\tilde{\mathcal{D}}_{G}$ are small allows us to form the classifying $G$-spaces $B h \tilde{\mathcal{D}}_{G}$ and $B \tilde{\mathcal{D}}_{G}$ as the geometric realizations of two-sided bar constructions, as in $[\mathbf{1}, 20.2]$ or [14]. Since categories equivalent over $\mathcal{O}_{G}$ give $G$-homotopy equivalent classifying spaces, $B h \tilde{\mathcal{D}}_{G}$ is, up to $G$-homotopy, independent of the choice of $h \tilde{\mathcal{D}}_{G}$. The corresponding statement about $B \tilde{\mathcal{D}}_{G}$ follows from the next theorem.

By [14] the space $B \tilde{\mathcal{D}}_{G}$ classifies $G$-fibrations with fibers modeled on the objects of $\mathcal{D}_{G}$, as long as $\tilde{\mathcal{D}}_{G}$ contains at least one object from each homotopy type of homotopy $G$-covering spaces over orbits. In other words, $B \tilde{\mathcal{D}}_{G}$ classifies homotopy $G$-covering spaces with fibers restricted as in the definition of $\tilde{\mathcal{D}}_{G}$. Similarly, $B \tilde{\mathcal{D}}_{G}^{s}$ classifies $G$-covering spaces whose fibers are restricted in cardinality. So, we have the following result.

Theorem 7.2. $\mathcal{E} \tilde{\mathcal{C}}(X) \cong\left[X, B \tilde{\mathcal{D}}_{G}^{s}\right]_{G}$ and $\mathcal{E} \tilde{\mathcal{C}}_{h}(X) \cong\left[X, B \tilde{\mathcal{D}}_{G}\right]_{G}$.

On the other hand, the following is a special case of $[\mathbf{1}, 24.1]$.

Theorem 7.3. $\mathcal{E} \tilde{\mathcal{R}}(X) \cong\left[X, B h \tilde{\mathcal{D}}_{G}^{s}\right]_{G}$ and $\mathcal{E} \tilde{\mathcal{R}}_{h}(X) \cong\left[X, B h \tilde{\mathcal{D}}_{G}\right]_{G}$.

Now, we know that $h \tilde{\mathcal{D}}_{G}^{s}=\tilde{\mathcal{D}}_{G}^{s}$, so $B h \tilde{\mathcal{D}}_{G}^{s}=B \tilde{\mathcal{D}}_{G}^{s}$. From Theorem A and the two theorems above we get:

Corollary 7.4. For any $G$-space $X$ of the $G$-homotopy type of a $G$-CW complex, we have $\left[X, B h \tilde{\mathcal{D}}_{G}\right]_{G} \cong\left[X, B \tilde{\mathcal{D}}_{G}\right]_{G}$.

In fact, we can show more directly that $B h \tilde{\mathcal{D}}_{G}$ and $B \tilde{\mathcal{D}}_{G}$ are weakly $G$-equivalent: It follows from Lemmas 2.3 and 3.8 that $B \pi_{0}: B \tilde{\mathcal{D}}_{G} \rightarrow B \tilde{\mathcal{F}}_{G}$ and $B \pi_{0}: B h \tilde{\mathcal{D}}_{G} \rightarrow$ $B \tilde{\mathcal{F}}_{G}$ are both weak $G$-equivalences. Hence, $B \tilde{\mathcal{D}}_{G}$ and $B h \tilde{\mathcal{D}}_{G}$ are weakly $G$-equivalent.

\section{Examples}

To illustrate the preceding results we classify the covering spaces and homotopy covering spaces over the one-point compactifications of representations of $\mathbb{Z} / 2$ and $\mathbb{Z} / 3$. We begin with $\mathbb{Z} / 3$, which is technically simpler.

8.1. $G=\mathbb{Z} / 3$

The orbit category of $G$ has two objects and maps as in the following diagram.

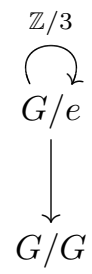


Let $V$ be a finite-dimensional real representation of $G=\mathbb{Z} / 3$. The case $V=0$ being uninteresting, we are left with four cases to consider.

Case 1: $V^{G}=0$ and $|V| \geqslant 2$

The one-point compactification of $V, S^{V}$, has two fixed points and is nonequivariantly simply-connected. We work with a skeleton of its fundamental groupoid having three objects: $N$ (north pole) and $S$ (south pole) over $G / G$, and $E$ (equator) over $G / e$. We can picture this category as follows.

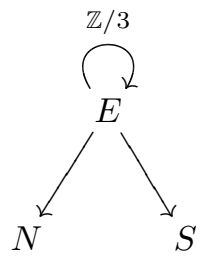

A discrete action, $A$, of the fundamental groupoid is then specified by giving two sets, $A(N)$ and $A(S)$, and a $\mathbb{Z} / 3$-set $A(E)$, with maps $A(N) \rightarrow A(E)^{\mathbb{Z} / 3}$ and $A(S) \rightarrow$ $A(E)^{\mathbb{Z} / 3}$.

A discrete action $A$ is strictly discrete if $A(N)=A(E)^{\mathbb{Z} / 3}$ and $A(S)=A(E)^{\mathbb{Z} / 3}$. In other words, a strictly discrete action is determined by the $\mathbb{Z} / 3$-set $A(E)$.

Any discrete action is the disjoint union of actions irreducible under disjoint union. It's clear that the decomposition into irreducible actions corresponds to the decomposition of $A(E)$ into orbits.

Thus, there are exactly two irreducible strictly discrete actions, corresponding to $A(E)=\mathbb{Z} / 3$ and $A(E)=*=(\mathbb{Z} / 3) /(\mathbb{Z} / 3)$. The corresponding $G$-covering spaces are the projection $\mathbb{Z} / 3 \times S^{V} \rightarrow S^{V}$ and the identity $S^{V} \rightarrow S^{V}$, respectively. In general, the $G$-covering space corresponding to a strictly discrete action $A$ is $A(E) \times S^{V} \rightarrow S^{V}$.

As for the possible homotopy covering spaces, the only interesting irreducible actions left to discuss are those with $A(E)=*$. The sets $A(N)$ and $A(S)$ can be arbitrary. The corresponding homotopy $G$-covering space $X$ is such that $X \rightarrow S^{V}$ is a nonequivariant homotopy equivalence. The fiber over $N$ is a contractible space with an action of $\mathbb{Z} / 3$ whose $\mathbb{Z} / 3$-fixed set is homotopy equivalent to $A(N)$, and likewise for the fiber over $S$.

When $A(N)$ and $A(S)$ have at least one point each, we can give an explicit construction of such a homotopy $G$-covering space as follows. Take

$$
X=\left(\bigvee_{|A(N)|-1} S^{\infty V}\right) \cup S^{V} \cup\left(\bigvee_{|A(S)|-1} S^{\infty V}\right)
$$

where $S^{\infty V}=\operatorname{colim}_{n} S^{n V}$, the first union identifies the wedge point with the north pole, and the second union identifies the wedge point with the south pole. The map $X \rightarrow S^{V}$ that takes each wedge to the corresponding pole is a $G$-quasifibration. The associated $G$-fibration is then a homotopy $G$-covering space corresponding to $A$.

Finally, the identity map $S^{V} \rightarrow S^{V}$ is a $G$-covering space whose restriction to each component of each fixed set is the simply-connected covering. 


\section{Case 2: $V=\mathbb{R}$ (with trivial $G$-action)}

In this case the fundamental groupoid has a skeleton with two objects, $S$ (stationary) and $E$, with maps as shown in the following diagram.

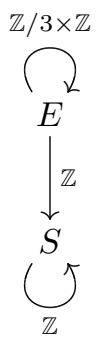

A discrete action of the fundamental groupoid is specified by a $\mathbb{Z}$-set $A(S)$, a $\mathbb{Z} / 3 \times \mathbb{Z}$ set $A(E)$, and a $\mathbb{Z}$-map $A(S) \rightarrow A(E)^{\mathbb{Z} / 3}$. The strictly discrete representations are those in which $A(S)=A(E)^{\mathbb{Z} / 3}$.

An action is irreducible when $A(E)=(\mathbb{Z} / 3 \times \mathbb{Z}) / H$ for some subgroup $H \subset$ $\mathbb{Z} / 3 \times \mathbb{Z}$. We have the following possible cases.

(a) $H=0 \times n \mathbb{Z}$ for some $n \geqslant 0$. In this case, $A(E)=\mathbb{Z} / 3 \times \mathbb{Z} / n$ and $A(S)=\emptyset$, so $A$ is strictly discrete. The corresponding $G$-covering space is the product of $\mathbb{Z} / 3$ with the $n$-fold covering space of $S^{1}$ (or the line, if $n=0$ ).

(b) $H$ is the subgroup generated by $(1, n)$ or $(2, n)$, with $n>0$. In this case, $A(E)=\mathbb{Z} /(3 n)$ with $\mathbb{Z} / 3$ acting by addition of $n$ (when $H$ is generated by $(1, n)$ ) or subtraction of $n$ (when $H$ is generated by $(2, n)$ ). Again, $A(S)=\emptyset$ and $A$ is strictly discrete. The corresponding $G$-covering space is the $3 n$-fold covering of the circle, with $\mathbb{Z} / 3$ acting on the total space by rotation by $\pm 2 \pi / 3$.

(c) $H=\mathbb{Z} / 3 \times n \mathbb{Z}$ for some $n \geqslant 0$. In this case, $A(E)=\mathbb{Z} / n$ with trivial $\mathbb{Z} / 3$-action and $A(S)$ can be any $\mathbb{Z}$-set mapping to $\mathbb{Z} / n$. When $A(S)=\mathbb{Z} / n, A$ is strictly discrete and the corresponding $G$-covering space is the $n$-fold covering of the circle with trivial $\mathbb{Z} / 3$-action.

In general, let $X$ be a homotopy $G$-covering space corresponding to $A$. Nonequivariantly, $X \rightarrow S^{1}$ is equivalent to the $n$-fold covering. However, $X^{\mathbb{Z} / 3} \rightarrow$ $S^{1}$ is equivalent to a disjoint union of $k n$-fold coverings and simply-connected coverings, corresponding to the decomposition of $A(S)$ as a disjoint union of $\mathbb{Z}$-orbits over $\mathbb{Z} / n$. There is no obvious, simple construction of such a space.

The $G$-covering $\mathbb{R} \rightarrow S^{1}$, with trivial $G$-action, is a covering whose restriction to each component of each fixed set is the simply-connected cover. 
Case 3: $\left|V^{G}\right|=1$ and $|V|>1$

The fundamental groupoid of $S^{V}$ again has a skeleton with two objects, $S$ and $E$, with maps as shown in the following diagram.

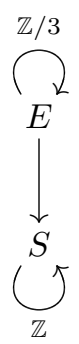

A discrete action of the fundamental groupoid is specified by a $\mathbb{Z}$-set $A(S)$, a $\mathbb{Z} / 3$ set $A(E)$, and a function $A(S) / \mathbb{Z} \rightarrow A(E)^{\mathbb{Z} / 3}$. The strictly discrete representations are those in which $\mathbb{Z}$ acts trivially on $A(S)$ and $A(S)=A(E)^{\mathbb{Z} / 3}$.

There are exactly two irreducible strictly discrete representations, corresponding to $A(E)=\mathbb{Z} / 3$ and $A(E)=*$. In the former case $A(S)=\emptyset$; the corresponding $G$-covering space is $\mathbb{Z} / 3 \times S^{V} \rightarrow S^{V}$. In the latter case $A(S)=*$; the corresponding $G$-covering space is the identity map $S^{V} \rightarrow S^{V}$. In general, the $G$-covering space corresponding to a strictly discrete action $A$ is $A(E) \times S^{V} \rightarrow S^{V}$.

The remaining irreducible discrete representations have $A(E)=*$ and $A(S)$ an arbitrary $\mathbb{Z}$-set. A corresponding homotopy $G$-covering space $X \rightarrow S^{V}$ is nonequivariantly equivalent to the identity map $S^{V} \rightarrow S^{V}$, while the fixed-point map $X^{G} \rightarrow S^{1}$ is an arbitrary disjoint union of covering spaces of the circle. In general there is no obvious, simple construction of such a space.

The homotopy $G$-covering space corresponding to the representation with $A(E)=$ * and $A(S)=\mathbb{Z}$ has the property that its restriction to each component of each fixed set is equivalent to the simply-connected cover. In this case, no $G$-covering space has this property.

Case 4: $\left|V^{G}\right| \geqslant 2$

The fundamental groupoid of $S^{V}$ is now equivalent to the orbit category:

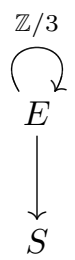

A discrete action of the fundamental groupoid is specified by a set $A(S)$, a $\mathbb{Z} / 3$-set $A(E)$, and a function $A(S) \rightarrow A(E)^{\mathbb{Z} / 3}$. The strictly discrete representations are those in which $A(S)=A(E)^{\mathbb{Z} / 3}$.

Thus, there are two irreducible strictly discrete actions, one with $A(E)=\mathbb{Z} / 3$ and the other with $A(E)=*$. The corresponding covering spaces are $\mathbb{Z} / 3 \times S^{V} \rightarrow S^{V}$ and the identity $S^{V} \rightarrow S^{V}$. In general, the $G$-covering space corresponding to a 
strictly discrete representation $A$ is the product $A(E) \times S^{V} \rightarrow S^{V}$.

If $A(E)=*$ and $A(S)$ is any set, a corresponding homotopy $G$-covering space is the product $F \times S^{V} \rightarrow S^{V}$ where $F$ is a nonequivariantly contractible $G$-space with $F^{G}$ equivalent to $A(S)$. Models of such spaces $F$ are well-known. For $|A(S)| \geqslant 2$ we can use a wedge of infinite spheres as in Case 1 above.

As in Case 1, the identity map $S^{V} \rightarrow S^{V}$ is the $G$-covering whose restriction to each component of each fixed set is the simply-connected cover.

\section{2. $G=\mathbb{Z} / 2$}

The orbit category of $G=\mathbb{Z} / 2$ has two objects and maps as in the following diagram.

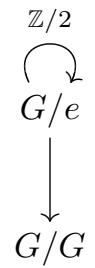

All of the cases we considered for $G=\mathbb{Z} / 3$ occur again for $G=\mathbb{Z} / 2$, with the same conclusions. However, we have one additional and particularly interesting case:

New Case: $V^{G}=0$ and $|V|=1$

This is the case where $V$ is the real line with the nontrivial linear action of $G$. A skeleton of the fundamental groupoid of $S^{V}$ has three objects, the poles $N$ and $S$ over $G / G$ and the equator $E$ over $G / e$. To describe the maps in this category we need to be very explicit about choices. Picture $S^{V}$ as a circle with the north pole at the top, the south pole at the bottom, and the point $E$ being the equatorial point on the right. In the fundamental groupoid, $E$ has a self-map over the identity we shall call $\sigma$, represented by the loop going once around the circle counterclockwise. It has another self-map we shall call $\tau$, over the nontrivial self-map of $G / e$, represented by the path going halfway around the circle counterclockwise, from $E$ to its translate on the left. These two maps generate the group of self-maps of $E$, which is the infinite dihedral group

$$
D_{\infty}=\left\langle\sigma, \tau \mid \tau^{2}=e, \tau \sigma=\sigma^{-1} \tau\right\rangle .
$$

Let $\pi_{N}: E \rightarrow N$ be the map represented by the shortest path from $E$ to $N$. The set of all maps from $E$ to $N$ is the orbit of $\pi_{N}$ under the (right) action of $D_{\infty}$. The stabilizer of $\pi_{N}$ is $\langle\tau\rangle$, so we can identify the set of maps from $E$ to $N$ with the orbit $\langle\tau\rangle \backslash D_{\infty}$. A similar analysis, using the shortest path $\pi_{S}$ from $E$ to $S$, allows us to identify the set of maps from $E$ to $S$ with $\langle\sigma \tau\rangle \backslash D_{\infty}$. The fundamental groupoid 
can therefore be pictured as follows.

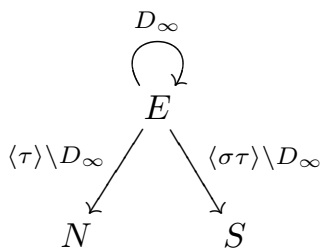

If $A$ is a discrete action of the fundamental groupoid, evaluation determines two $D_{\infty}$-functions

$$
A(N) \times \operatorname{map}(E, N)=A(N) \times\langle\tau\rangle \backslash D_{\infty} \rightarrow A(E)
$$

and

$$
A(S) \times \operatorname{map}(E, S)=A(S) \times\langle\sigma \tau\rangle \backslash D_{\infty} \rightarrow A(E) .
$$

(Again, note that all $D_{\infty}$ actions are from the right.) Conversely, any two such $D_{\infty}$-functions determine a discrete action. Notice that, since $\pi_{N}$ is fixed by $\tau$, the image of $A(N)$ in $A(E)$ under $\pi_{N}^{*}$ must be fixed by $\tau$. Similarly, the image of $A(S)$ under $\pi_{S}^{*}$ must be fixed by $\sigma \tau$. The action $A$ is strictly discrete if and only if $\pi_{N}^{*}$ is the inclusion of the $\tau$-fixed points and $\pi_{S}^{*}$ is the inclusion of the $\sigma \tau$-fixed points.

We can now show that there is no homotopy $G$-covering space of $S^{V}$ whose fixed sets are equivalent over each component to the simply-connected cover. If there were such, the corresponding action $A$ would have $A(E) \cong \mathbb{Z}$ with the subgroup $\mathbb{Z}=\langle\sigma\rangle \subset D_{\infty}$ acting in the obvious way. There are, up to isomorphism, two such actions of $D_{\infty}$, namely $\langle\tau\rangle \backslash D_{\infty}$ and $\langle\sigma \tau\rangle \backslash D_{\infty}$. (In the former $\tau$ acts by negation on $\mathbb{Z}$, while in the latter it acts by negation plus 1.) We would also have $A(N)$ and $A(S)$ each consisting of a single point. However, if $A(E) \cong\langle\tau\rangle \backslash D_{\infty}$ then $A(E)$ has no $\sigma \tau$-fixed point, so there is no place for $A(S)$ to go, hence no such action $A$ is possible. On the other hand, if $A(E) \cong\langle\sigma \tau\rangle \backslash D_{\infty}$, then $A(E)$ has no $\tau$-fixed point, so there is no place for $A(N)$ to go. Therefore, there is no such action, and there is no such homotopy $G$-covering space.

We now consider what homotopy $G$-covering spaces do exist, by considering the possible irreducible actions. We consider various cases based on the possibilities for the orbit $A(E)$.

(a) $A(E) \cong e \backslash D_{\infty}$. In this case we must have $A(N)$ and $A(S)$ both empty, so the action is strict. The corresponding covering space is the $\mathbb{Z} / 2$-map $\mathbb{Z} / 2 \times \mathbb{R} \rightarrow S^{V}$ extending the nonequivariant covering $\mathbb{R} \rightarrow S^{V}$ (where $\mathbb{Z} / 2$ acts trivially on $\mathbb{R}$ ).

(b) $A(E) \cong\left\langle\sigma^{n}\right\rangle \backslash D_{\infty} \cong D_{2 n}$ for some $n \geqslant 1$. Again, both $A(N)$ and $A(S)$ must be empty, so the action is strict. The corresponding covering space is the $\mathbb{Z} / 2$-map $\mathbb{Z} / 2 \times S^{1} \rightarrow S^{V}$ extending the nonequivariant $n$-fold covering $S^{1} \rightarrow S^{V}$ (where $\mathbb{Z} / 2$ acts trivially on $\left.S^{1}\right)$.

(c) $A(E) \cong\left\langle\sigma^{n} \tau\right\rangle \backslash D_{\infty} \cong \mathbb{Z}$ for $n=0$ or 1 . (Other integer values of $n$ yield orbits isomorphic to one of these two cases.) If $A(E) \cong\langle\tau\rangle \backslash D_{\infty}$ then $\tau$ has one fixed point in $A(E)$ but $\sigma \tau$ has none. Therefore, $A(N)$ can be any set but $A(S)$ must be empty. When $A(N)$ is a single point, with $\pi_{N}^{*}$ taking it to the $\tau$-fixed 
point in $A(E), A$ is strictly discrete. The corresponding $G$-covering space is the simply-connected cover $V \rightarrow S^{V}$ taking the fixed-point (the origin) in $V$ to the north pole of $S^{V}$. Note that the action of $G$ on the fiber over the south pole is free.

Continuing to assume that $A(E) \cong\langle\tau\rangle \backslash D_{\infty}$, but now allowing $A(N)$ to be any set, a corresponding homotopy $G$-covering space can be obtained from the $G$-covering space $V \rightarrow S^{V}$ above by crossing the total space $V$ with a $G$-space $F$ which is nonequivariantly contractible and has $F^{G} \simeq A(N)$. Constructions of such spaces are well known.

On the other hand, if $A(E) \cong\langle\sigma \tau\rangle \backslash D_{\infty}$, we must have $A(N)$ empty and $A(S)$ can be any set. If $A(S)$ is a single point, the corresponding $G$-covering space is the simply-connected cover $V \rightarrow S^{V}$ taking the fixed-point in $V$ to the south pole. If $A(S)$ is any set, a corresponding homotopy $G$-covering space is $V \times F \rightarrow$ $S^{V}$ where $F$ is a nonequivariantly contractible $G$-space with $F^{G} \simeq A(S)$.

(d) $A(E) \cong\left\langle\sigma^{n}, \tau\right\rangle \backslash D_{\infty} \cong \mathbb{Z} / n$ or $A(E) \cong\left\langle\sigma^{n}, \sigma \tau\right\rangle \backslash D_{\infty} \cong \mathbb{Z} / n$, for some $n \geqslant 1$. When $n$ is odd these two orbits are isomorphic $D_{\infty}$-sets, but when $n$ is even they are not. Suppose first that $n$ is odd. Then $\tau$ acts on $A(E) \cong \mathbb{Z} / n$ by negation and there is a single fixed point, 0 . On the other hand, $\sigma \tau$ acts by negation followed by addition of 1 , and there is a single fixed point for this action as well, namely $(n+1) / 2$. Thus, both $A(N)$ and $A(S)$ may be nonempty. $A$ is strictly discrete when both $A(N)$ and $A(S)$ are single points. The corresponding $G$-covering space is the $n$-fold cover $S^{V} \rightarrow S^{V}$. If we assume that the north pole maps to the north pole, then, because $n$ is odd, the south pole maps to the south pole. Continuing to assume that $n$ is odd, if $A$ is not strictly discrete then $A(N)$ and $A(S)$ may be arbitrary sets. A corresponding homotopy $G$-covering space $X \rightarrow S^{V}$ is nonequivariantly equivalent to the $n$-fold cover. The action of $G$ on the fiber over the north pole permutes the components as $\tau$ permutes $\mathbb{Z} / n$, but the $G$-fixed set is equivalent to $A(N)$. Similarly, the fiber over the south pole has $G$-fixed set equivalent to $A(S)$. When $A(N)$ and $A(S)$ have at least one point each, we can give an explicit construction of such a space as follows. Let $F_{N}$ and $F_{S}$ be nonequivariantly contractible pointed $G$-spaces with $F_{N}^{G} \simeq A(N)$ and $F_{S}^{G} \simeq A(S)$. Take $X=F_{N} \cup S^{V} \cup F_{S}$ where the first union identifies the basepoint with the north pole and the second identifies the basepoint with the south pole. The map $X \rightarrow S^{V}$ obtained by mapping $F_{N}$ to the north pole, $F_{S}$ to the south pole, and $S^{V}$ to itself by the $n$-fold cover, is a $G$-quasifibration. The associated $G$-fibration is then a homotopy $G$-covering space corresponding to $A$.

Now consider the case where $n$ is even and suppose that $A(E) \cong\left\langle\sigma^{n}, \tau\right\rangle \backslash D_{\infty} \cong$ $\mathbb{Z} / n$. $\tau$ acts on this orbit by negation, so has two fixed points, 0 and $n / 2$. $\sigma \tau$ acts by negation followed by addition of 1 , and has no fixed points. Therefore, $A(S)$ must be empty but $A(N)$ can be any set. The function $\pi_{N}^{*}$ maps $A(N)$ into the two-point set $\{0, n / 2\}$, so $A(N)$ is the disjoint union of the two sets $A_{0}=\left(\pi_{N}^{*}\right)^{-1}(0)$ and $A_{1}=\left(\pi_{N}^{*}\right)^{-1}(n / 2) . A$ is strictly discrete when $A_{0}$ and $A_{1}$ are both single points. The corresponding $G$-covering space is the $n$-fold covering $S^{V} \rightarrow S^{V}$ that maps both the north and south poles to the north 
pole.

Continuing to assume that $n$ is even and that $A(E) \cong\left\langle\sigma^{n}, \tau\right\rangle \backslash D_{\infty} \cong \mathbb{Z} / n$, let $A_{0}$ and $A_{1}$ be arbitrary sets. A corresponding homotopy $G$-covering space $X \rightarrow S^{V}$ is nonequivariantly equivalent to the $n$-fold cover. The action of $G$ on the fiber over the north pole permutes the components as $\tau$ permutes $\mathbb{Z} / n$, so carries two of the components to themselves. The fixed set in one component is equivalent to $A_{0}$ while the fixed set in the other component is equivalent to $A_{1}$. The action of $G$ on the fiber over the south pole leaves no component invariant. An explicit model for such a homotopy $G$-covering space can be constructed in a manner similar to the model constructed above for $n$ odd.

When $n$ is even and $A(E) \cong\left\langle\sigma^{n}, \sigma \tau\right\rangle \backslash D_{\infty} \cong \mathbb{Z} / n$, we can repeat the discussion above, reversing the roles of the north and south poles.

\section{Twisted coefficients}

In this section we restrict to a finite group $G$.

In [2] we gave a construction of ordinary equivariant homology and cohomology with twisted (or local) coefficients. The ad hoc construction given there can be clarified and reformulated in the light of the results of the preceding sections.

It is well-known that the appropriate coefficient systems for $R O(G)$-graded ordinary (co)homology are the Mackey functors, which are abelian group-valued functors defined on $\hat{\mathcal{O}}_{G}$, the stable orbit category of $G$. Analogously, the appropriate twisted coefficient systems are abelian group-valued functors defined on the stable fundamental groupoid, as in [2]. The following definition is appropriate for finite groups but not for compact Lie groups:

Definition 9.1. Let $X$ be a $G$-space. The stable fundamental groupoid of $X$ is the category $\hat{\Pi}_{G} X$ defined as follows. Its objects are those of $\Pi_{G} X$. The set of morphisms from $x$ to $y$ is the free abelian group on the set of equivalence classes of diagrams $x \leftarrow a \rightarrow y$ in $\Pi_{G} X$. The diagram $x \leftarrow a \rightarrow y$ is equivalent to the diagram $x \leftarrow a^{\prime} \rightarrow y$ if there is an isomorphism $a \rightarrow a^{\prime}$ in $\Pi_{G} X$ making the following diagram commute.

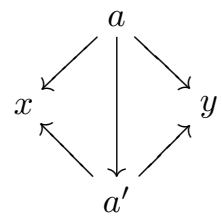

To compose a generator $x \leftarrow a \rightarrow y$ with a generator $y \leftarrow b \rightarrow z$, we first consider the following diagram of finite $G$-sets, where $P$ is the pullback in the middle square.

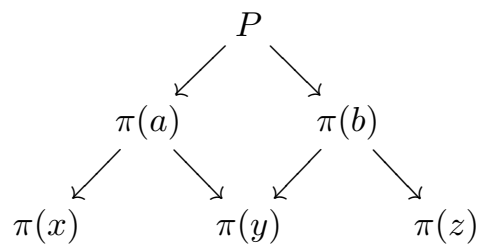


Now we decompose $P$ into a disjoint union of orbits. If $Q \subset P$ is an orbit, we can find an object $q$ of $\Pi_{G} X$ and maps $x \leftarrow q \rightarrow z$ covering $\pi(x) \leftarrow Q \rightarrow \pi(z)$ (this uses the source lifting and divisibility properties discussed in [1]). This gives a well-defined generator of the group of morphisms from $x$ to $z$, and we define the composite map to be the sum of these generators.

There is a canonical functor $\hat{\pi}: \hat{\Pi}_{G} X \rightarrow \hat{\mathcal{O}}_{G}$ extending $\pi$.

The twisted coefficient systems we use for ordinary homology are the covariant abelian group-valued functors on $\hat{\Pi}_{G} X$. The twisted coefficient systems for ordinary cohomology are the contravariant abelian group-valued functors on $\hat{\Pi}_{G} X$. On the other hand, these types of coefficient systems can be identified using the evident self-duality of $\hat{\Pi}_{G} X$. (Note that this is true for finite groups, but the analogous statement for compact Lie groups is not true.)

Nonequivariantly, twisted coefficients can be introduced into (co)homology by replacing the $\mathbb{Z}$-module $C_{*}(X)$ of cellular chains by the $\mathbb{Z} \pi_{1}(X)$-module $C_{*}(\tilde{X})$ of cellular chains of the simply-connected cover. Equivariantly, the process is not that simple. We replace $\mathbb{Z} \pi_{1}(X)$ with $\hat{\Pi}_{G} X$, but we have no obvious analogue of $\tilde{X}$, given the counterexample in the preceding section showing that, in general, there is no simply-connected homotopy $G$-covering space. What we can do is the following.

Construction 9.2. Let $X$ be a $G$-space whose fixed sets are all semi-locally simply connected. We first define a covariant functor over $\mathcal{O}_{G}$,

$$
\tilde{X}^{*}: \Pi_{G} X \rightarrow \operatorname{map}\left(\Pi_{G}(X \times-), \text { Set }\right)=\mathcal{A}_{h}(X \times-) .
$$

Here $\operatorname{map}\left(\Pi_{G}(X \times-)\right.$, Set $)$ denotes the category over $\mathcal{O}_{G}$ whose objects are the contravariant functors $A: \Pi_{G}(X \times G / H) \rightarrow$ Set. A morphism from $A$ to $B: \Pi_{G}(X \times$ $G / K) \rightarrow$ Set is a pair $(\sigma, \omega)$ where $\sigma: G / H \rightarrow G / K$ is a $G$-map and $\omega: A \rightarrow B \circ \sigma_{*}$ is a natural isomorphism. The functor $\tilde{X}^{*}$ is defined by

$$
\tilde{X}^{*}(x: G / H \rightarrow X)((y, \tau): G / K \rightarrow X \times G / H)=\Pi_{G} X(y, x)_{\tau},
$$

the set of maps in $\Pi_{G} X(y, x)$ that map to $\tau$ in $\mathcal{O}_{G}$. Note that $\tilde{X}(x)$ is a discrete action of $\Pi_{G} X(X \times G / H)$, i.e., an object of $\mathcal{A}_{h}(X \times G / H)$. Applying $\Psi: \mathcal{A}_{h}(X \times$ $G / H) \rightarrow \mathcal{C}_{h}(X \times G / H)$ we get a functor

$$
\tilde{X}: \Pi_{G} X \rightarrow \mathcal{C}_{h}(X \times-) .
$$

Here $\mathcal{C}_{h}(X \times-)$ is the category over $\mathcal{O}_{G}$ whose objects are the pairs $(G / H, p)$, where $p: Y \rightarrow X \times G / H$ is a homotopy $G$-covering space. A morphism from $(G / H, p)$ to $(G / K, q)$ is a pair $(\sigma, f)$ where $\sigma: G / H \rightarrow G / K$ and $f: p \rightarrow q$ is a fiberwise homotopy equivalence covering $1 \times \sigma: X \times G / H \rightarrow X \times G / K$.

We now extend to a functor

$$
\tilde{X}: \hat{\Pi}_{G} X \rightarrow h \hat{\mathcal{C}}_{h}(X \times-),
$$

where $\hat{\mathcal{C}}_{h}(X \times-)$ has the same objects as $\mathcal{C}_{h}(X \times-)$, but a map from $Y \rightarrow X \times G / H$ to $Z \rightarrow X \times G / K$ is given by a $G$-map $\sigma: G / H \rightarrow G / K$ and a map $\Sigma^{V} Y^{+} \rightarrow \Sigma^{V} Z^{+}$ 
such that the following diagram commutes.

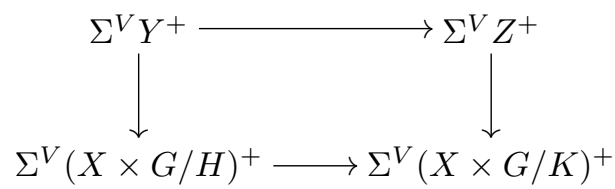

We identify stably equivalent maps in the usual way. In $h \hat{\mathcal{C}}_{h}(X \times-)$ we identify fiberwise homotopic maps. To extend $\tilde{X}$ to $\hat{\Pi}_{G} X$ it suffices to define it on a "transfer" map $x \leftarrow y=y$. Let $(\sigma, \omega): y \rightarrow x$ be the given map in $\Pi_{G} X$, where $\sigma: G / K \rightarrow G / H$. Let $V$ be a representation of $G$ in which $G / K$ embeds, and let $i: G / K \rightarrow V$ be an embedding. This gives the following diagram.

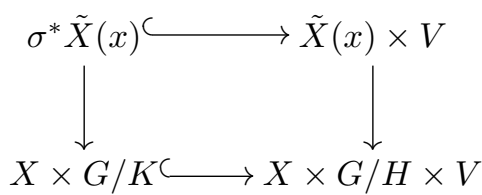

Taking collapse maps then gives us:

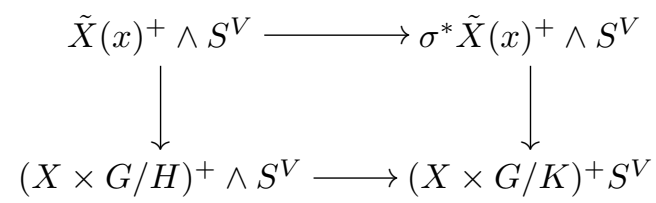

Composing with a fiberwise equivalence $\sigma^{*} \tilde{X}(x) \rightarrow \tilde{X}(y)$ inverse to the fiberwise equivalence induced by $\tilde{X}$, we get a well-defined stable fiberwise homotopy class of maps from $\tilde{X}(x) \rightarrow \tilde{X}(y)$.

Now suppose that $X$ is given a suitable cellular filtration $X^{0} \subset X^{1} \subset \cdots \subset X$. This might be a $G$-CW filtration in the sense of Bredon or a $G$-CW $(V)$ or $G$-CW $(\gamma)$ filtration as in [15] or [2]. This filtration induces a filtration of $\tilde{X}$ which we can use to define the cellular chains, a covariant functor

$$
C_{*}(\tilde{X}): \hat{\Pi}_{G} X \rightarrow \mathrm{Ab}
$$

as the appropriate stable homotopy groups of the filtration quotients. Using the self-duality of $\hat{\Pi}_{G} X$ we can also consider $C_{*}(\tilde{X})$ to be a contravariant functor. 
Now, if $A$ is a covariant twisted coefficient system, we define the twisted $G$ homology of $X$ with coefficients in $A$ to be the homology of the chain complex $C_{*}(\tilde{X}) \otimes_{\hat{\Pi}_{G} X} A$. Similarly, if $A$ is a contravariant twisted coefficient system, we define the twisted $G$-cohomology of $X$ with coefficients in $A$ to be the homology of the

cochain complex $\operatorname{Hom}_{\hat{\Pi}_{G} X}\left(C_{*}(\tilde{X}), A\right)$. For an application to equivariant Poincaré duality and simple Poincaré duality, see $[\mathbf{2}]$ and $[\mathbf{3}]$.

\section{References}

[1] S. R. Costenoble, J. P. May, and S. Waner, Equivariant orientation theory, Homology, Homotopy and Applications 3 (2001), no. 2, 265-339.

[2] S. R. Costenoble and S. Waner, Equivariant Poincaré duality, Michigan Math. J. 39 (1992), 325-351.

[3] _ Equivariant simple Poincaré duality, Michigan Math. J. 40 (1993), 577-604.

[4] A. D. Elmendorf, Systems of fixed point sets, Trans. Amer. Math. Soc. 277 (1983), 275-284.

[5] - Actions, functors and the bar construction, Cahiers Topologie Géom. Différentielle Catégoriques XXVI (1985), 329-338.

[6] A. Grothendieck, Rêvetements étale et groupe fondemental (SGA1), Lecture Notes in Math., vol. 224, Springer-Verlag, 1971.

[7] J. P. May, Classifying spaces and fibrations, Mem. Amer. Math. Soc. 155 (1972).

[8] , Fibrewise localization and completion, Trans. Amer. Math. Soc. 258 (1980), 127-146.

[9] R. S. Palais, The classification of $G$-spaces, Mem. Amer. Math. Soc. 36 (1960).

[10] R. Schön, Fibrations over a CWh-base, Proc. Amer. Math. Soc. 62 (1977), $165-166$.

[11] J. D. Stasheff, A classification theorem for fibre spaces, Topology 2 (1963), 239-246.

[12] T. tom Dieck, Transformation groups, Studies in Math., vol. 8, Walter de Gruyter, 1987.

[13] S. Waner, Equivariant homotopy theory and Milnor's theorem, Trans. Amer. Math. Soc. 258 (1980), 351-368. 
[14] Equivariant classifying spaces and fibrations, Trans. Amer. Math. Soc. 258 (1980), 385-405.

[15],$G-C W(V)$ complexes and $R O(G)$-graded cohomology, Equivariant Homotopy and Cohomology Theory, CBMS Regional Conference Series in Mathematics, vol. 91, Amer. Math. Soc., 1996, pp. 89-96.

This article may be accessed via WWW at http://www.rmi.acnet.ge/hha/ or by anonymous ftp at

ftp://ftp.rmi.acnet.ge/pub/hha/volumes/2004/n1a23/v6n1a23.(dvi,ps,pdf)

Steven R. Costenoble Steven.R.Costenoble@Hofstra.edu

Department of Mathematics

103 Hofstra University

Hempstead, NY 11549-1030

Stefan Waner matszw@Hofstra.edu

Department of Mathematics

103 Hofstra University

Hempstead, NY 11549-1030 